\title{
Niveau de contamination et risques sanitaires liés à la consommation des produits maraîchers à Korhogo, Côte d'Ivoire
}

\author{
Rachelle Ida YAPO ${ }^{1,2^{*}}$, Marie Jeanne OHOU-YAO ${ }^{3}$, Raymond LIGBAN ${ }^{3}$, Parfait \\ $\mathrm{KOUAME}^{2}$, Véronique $\mathrm{MAMBO}^{1}$ et Bassirou BONFOH${ }^{2}$ \\ ${ }^{1}$ Université Nangui-Abrogoua, Côte d'Tvoire. 02 BP 801, Abidjan 02, Côte d'Ivoire. \\ ${ }^{2}$ Centre Suisse de Recherches Scientifiques en Côte d'Ivoire. 01 BP 1303, Abidjan 01, Côte d'Ivoire. \\ ${ }^{3}$ Université Jean Lorougnon Guédé. BP 150, Daloa, Côte d'Ivoire. \\ *Auteur correspondant, E-mail : yanis_ida@yahoo.fr; Tel. (+225) 0102486021
}

\section{REMERCIEMENTS}

La présente étude a bénéficié du financement du Programme d'Appui Stratégique à la Recherche Scientifique (PASRES).

\begin{tabular}{ccc}
\hline Received: 01-04-2021 & Accepted: 30-09-2021 & Published: 30-10-2021 \\
\hline
\end{tabular}

\section{RESUME}

L'usage non maîtrisé des pesticides a des effets sur la qualité des denrées alimentaires et la santé de la population. L'objectif de cette étude était d'évaluer l'exposition chimique associée à la consommation des produits maraîchers du département de Korhogo. A cet effet, des résidus de pesticides ont été extraits des produits maraîchers (Allium cepa, Lactuca sativa, Capsicum sp, Solanum lycopersicum et Phaseolus lunatus) par la méthode QuEchERS et analysés par un chromatographe en phase gazeuse couplé à un spectromètre de masse afin d'estimer les apports journaliers en pesticides et le quotient de danger pour les consommateurs. Ces analyses ont permis de détecter huit (08) pesticides à savoir le chlorpyriphos éthyle, le cyhalotrine, le cypermethrine, l'ortho-phenylphenol, le parathion, le spirodiclofen, le thiamethoxam et le zoxamid dans les produits maraîchers avec des concentrations qui variaient entre $0,43 \mu \mathrm{g} / \mathrm{kg}$ et 7,64 $\mu \mathrm{g} / \mathrm{kg}$. Les apports journaliers en pesticides fluctuaient entre $7,44.10^{-6} \mu \mathrm{g} / \mathrm{kg}$ de poids corporel/jour et $1173.10^{-6} \mu \mathrm{g} / \mathrm{kg}$ de poids corporel/jour, et le quotient de danger était compris entre $0,015.10^{-6}$ et $156,6.10^{-6}$ pour la consommation de ces produits maraîchers. Dans l'ensemble, le niveau de risque a été jugé acceptable pour l'homme car le quotient de danger était inférieur à 1 .

(C) 2021 International Formulae Group. All rights reserved.

Mots clés : Culture maraîchère, pesticides, exposition, évaluation de risque, méthode QuEchERS, Korhogo.

\section{Level of contamination and health risks associated with vegetable's consumption in Korhogo, Côte d'Ivoire}

\begin{abstract}
The uncontrolled use of pesticide has effects on foodstuffs quality and population's health. The main of this study was to assess exposure level of vegetable's consumers in Korhogo department. Also, pesticide residues were extracted from vegetable's samples (Allium cepa, Lactuca sativa, Capsicum sp, Solanum
\end{abstract}


lycopersicum and Phaseolus lunatus) using QuEchERS method and analyzed by gas chromatography with mass spectrometry (GC/MS). Eight (08) pesticides (chlorpyrifos ethyl, cyhalotrine, cypermethrin, orthophenylphenol, parathion, spirodiclofen, thiamethoxam and zoxamid) were detected in vegetable products with concentrations ranged from $0.43 \mu \mathrm{g} / \mathrm{kg}$ to $7.64 \mu \mathrm{g} / \mathrm{kg}$. Estimated daily intakes of pesticide residues ranged from $7.4 \times 10^{-6} \mu \mathrm{g} / \mathrm{kg}$ body weight/day to $4350.6 \times 10^{-6} \mu \mathrm{g} / \mathrm{kg}$ body weight $/ \mathrm{day}$, and the hazard index ranged between $0.015 \times 10^{-6}$ et $156.6 \times 10^{-6}$ for these food commodities. Overall, the level of risk was considered acceptable for humans because the hazard quotient was below 1 .

(C) 2021 International Formulae Group. All rights reserved.

Keywords: Vegetables, pesticides, risk assesssment, exposure, QuEchERS method, Korhogo.

\section{INTRODUCTION}

Les pesticides sont des agents chimiques ou biologiques capables de détruire les parasites ou de contrôler leur croissance et leur reproduction (Son, 2018 ; Dakuyo et al., 2020). En Côte d'Ivoire, avec la mise en œuvre de la politique sectorielle agricole qui vise, entre autres, l'amélioration de la productivité et de la compétitivité du secteur agricole, on assiste à une utilisation accrue des pesticides dans l'agriculture en général et la production maraîchère en particulier (Soro et al., 2018). Toutefois, des études ont souligné l'existence de mauvaises pratiques phytosanitaires notamment le non-respect des doses prescrites, des règles de protection et des consignes d'hygiène préconisés lors des traitements, ainsi que la mauvaise gestion des emballages des produits phytosanitaires (Soro et al., 2018 ; Mambe-Ani et al., 2019). En outre, l'utilisation intensive des pesticides chimiques et les mauvaises pratiques phytosanitaires sont associés à la contamination de l'environnement et ont de nombreux effets sur la santé humaine. Ces effets sont, à court terme, l'irritation de la peau et des muqueuses, les maux de tête, les nausées et de façon chronique les problèmes respiratoires, les troubles de la reproduction, les maladies endocriniennes, le cancer et la mort (Kpan et al., 2019; Mambe-Ani et al., 2019).

Face aux effets négatifs des pesticides sur la santé humaine, des inquiétudes apparaissent alors chez les consommateurs des produits maraîchers pourtant réputés pour leurs valeurs nutritionnelles (Dakuyo et al., 2020). Selon kapoor et al. (2013), l'analyse des résidus de pesticides dans les aliments est un moyen de déterminer le niveau d'exposition humaine à ces produits chimiques et, par conséquent, leurs risques potentiels pour la santé humaine.

C'est dans ce contexte que s'est inscrite cette étude qui s'intéressait aux produits maraîchers du département de Korhogo, l'une des grandes zones de production vivrière en Côte d'Ivoire. L'objectif de l'étude était d'évaluer le niveau d'exposition aux produits phytosanitaires des consommateurs des produits maraîchers cultivés dans cette zone. De façon spécifique, il s'est agi de déterminer les résidus des pesticides présents dans les produits maraîchers, d'évaluer la quantité de résidus de pesticides ingérés au cours de la consommation des produits maraîchers et de caractériser le risque d'exposition des consommateurs.

\section{MATÉRIEL ET MÉTHODES Zone d'étude}

L'étude a été conduite dans cinq (05) villages du département de Korhogo, à savoir Kassoumgbarga, Kouniguekaha, Kafiokaha, Nangasseréguè et Lavononkaha. Ces villages font partie des localités d'intervention du projet d'appui aux Petits Producteurs Maraîchers dans les régions de Savanes (PPMS). Le PPMS est un programme de réhabilitation et de reconstitution des capacités minimales de production mené en faveur des populations 
rurales vulnérables en vue de l'autosuffisance et de la sécurité alimentaire en Côte d'Ivoire.

Le département de Korhogo (Figure 1) est situé dans la région du Poro, au Nord de la Côte d'Ivoire, entre les $8^{\circ} 32$ et $10^{\circ} 20$ de latitude Nord et les $5^{\circ} 16$ et $6^{\circ} 16$ de longitude Ouest. Il couvre une superficie de $7402 \mathrm{~km}^{2}$ et compte environ 536851 habitants (RGPH, 2014). Le climat, de type soudanais, est marqué par deux grandes saisons, une pluvieuse qui s'étend de mai à octobre et, une sèche couvrant la période de novembre à avril. La pluviométrie annuelle varie entre $1000 \mathrm{~mm}$ et $1600 \mathrm{~mm}$ avec une moyenne annuelle de $1200 \mathrm{~mm}$ (SODEXAM, 2014 ; Yapo et al., 2016). Les sols du département de Korhogo sont ferralitiques avec un pH qui varie de 4,6 à 5,1 (Yapo et al., 2016). Sa végétation est majoritairement celle de la savane arborée qui se caractérise par des arbres et arbustes d'une hauteur comprise entre 8 et $12 \mathrm{~m}$ et disséminés avec une densité de couvert de l'ordre de 25 à $35 \%$ (Dro et al., 2013). Le relief y est faiblement ondulé et parsemé des inselbergs granitiques dont les altitudes varient entre 400 et $600 \mathrm{~m}$ (Dro et al., 2013). Les habitants de la région sont majoritairement agriculteurs et les principales cultures sont le coton, l'anacarde, l'igname, le maïs, l'arachide et les cultures maraîchères (Aloko-N'guessan et al., 2018) qui font l'objet de cette étude.

\section{Echantillonnage}

L'échantillonnage a porté sur le matériel végétal constitué de bulbes d'oignon (Allium cepa), de feuilles d'oignon, de laitues (Lactuca sativa), de piments (Capsicum sp), de tomates (Solanum lycopersicum) et de feuilles de haricot (Phaseolus lunatus) récoltés directement sur les différents sites de production. Ces produits font partie des espèces légumières les plus produites et les plus consommées dudit département (AlokoN'guessan et al., 2018).
Le plan de prélèvement a consisté à scinder chaque site de production en parcelle de $10 \times 10 \mathrm{~m}^{2}$ minimum. Sur chaque parcelle, un échantillon d'1 $\mathrm{kg}$ de chaque produit maraîcher a été prélevé de manière aléatoire de sorte à être représentatif du milieu. Au total, 63 échantillons de produits maraîchers, à savoir 18 échantillons de feuilles de haricot, 18 échantillons de feuilles d'oignon, 16 échantillons de piment, 6 échantillons de laitue et de 5 échantillons de bulbes d'oignon, ont été collectés. Les échantillons ont été emballés dans du papier aluminium et mis dans des sachets stomachers pour limiter les phénomènes d'oxydation. Ils ont été étiquetés et conservés à $4{ }^{\circ} \mathrm{C}$ dans une glacière pendant le transport, puis au congélateur à $-18{ }^{\circ} \mathrm{C}$ jusqu'au moment des analyses.

\section{Extraction et séparation des résidus de pesticides}

L'extraction des résidus de pesticides a été réalisée conformément à la méthode QuEchERS modifiée (Swarnam et Velmurugan, 2013). Ainsi, $10 \mathrm{~g}$ d'échantillon de produits maraîchers préalablement broyés ont été mis dans un tube à centrifuger de $50 \mathrm{ml}$ avec $10 \mathrm{ml}$ d'acétononitrile. Après une minute d'agitation au vortex, $4 \mathrm{~g}$ de magnésium sulfate anhydre $\left(\mathrm{MgSO}_{4}\right)$ et $1 \mathrm{~g}$ de chlorure de sodium $(\mathrm{NaCl})$ ont été ajoutés au mélange. L'ensemble a été centrifugé pendant 5 min à 3000 tours. Ensuite, $1 \mathrm{ml}$ de cet extrait a été prélevé et transféré dans un tube à centrifuger contenant $150 \mathrm{mg}$ de magnésium sulfate anhydre et 50 mg PSA (Primary Secondary Amine). La composition ainsi obtenue a été soumise à 5 min de centrifugation à 3000 tours. $0,5 \mathrm{ml}$ de l'extrait obtenu et $100 \mu \mathrm{l}$ de standard interne préparé dans l'acetonitrile à $0,25 \%$ d'acide formique ont été injectés dans une viale. Le conditionnement des extraits avant dosage a été fait dans l'hexane. 


\section{Dosage des résidus de pesticides}

La détection et la quantification des résidus de pesticides ont été faites à partir de l'injection de $1 \mu \mathrm{l}$ de l'extrait dans un chromatographe en phase gazeuse (GC) de type Agilent 7890A couplé à un spectromètre de masse (MS) de type Agilent 5975C équipé d'un détecteur sélectif quadripolaire. L'injection s'est réalisée en mode "splitless" avec un volume d'injection de $1 \mu \mathrm{l}$ et une température d'injecteur de $280{ }^{\circ} \mathrm{C}$. Le gaz vecteur était l'hélium de haute pureté (Air Liquide Alpha 2) avec un débit constant de 0,8 $\mathrm{ml} . \mathrm{min}^{-1}$. L'injecteur et le détecteur ont été respectivement maintenus à $280{ }^{\circ} \mathrm{C}$ et $300^{\circ} \mathrm{C}$.

L'identification des pesticides s'est faite par comparaison des temps de rétention obtenus en analysant une solution standard mixte de travail à $500 \mathrm{ppb}$ et en interrogeant la bibliothèque de spectres de masse du logiciel. Les concentrations des résidus de pesticides ont été déterminées selon la formule suivante (Yao et al., 2016) :

$$
C=\frac{\left(C_{i} \times V_{i} \times S_{e} \times V_{t}\right)}{\left(V_{e} \times S_{i} \times P\right)}
$$

Où $\mathrm{Ci}=$ concentration de la gamme étalon; $\mathrm{Vi}$ $=$ volume injecté de la gamme étalon $(\mu \mathrm{l}) ; \mathrm{Se}$ $=$ surface du pic de l'extrait $\left(\mathrm{cm}^{2}\right) ; \mathrm{Vt}=$ volume total de l'extrait $(\mathrm{ml}) ; \mathrm{Ve}=$ volume injecté de l'extrait $(\mu \mathrm{l}) ; \mathrm{Si}=$ surface du pic de la gamme étalon $\left(\mathrm{cm}^{2}\right) ; \mathrm{P}=$ poids de l'extrait (g).

\section{Evaluation du risque sanitaire}

L'évaluation du risque d'exposition aux résidus de pesticides tient compte des données de ces pesticides existant dans la littérature, de la teneur en résidus de pesticides de ces produits maraîchers et de leur fréquence de consommation. Ainsi, l'apport journalier estimatif de pesticide ingérable a été déterminé selon la formule suivante (Chowdhury et al., 2014) :

$$
A J E=\frac{(C \times Q \times F C \times F E)}{P}
$$

Où $\mathrm{AJE}=$ Apport journalier estimatif $(\mu \mathrm{g} / \mathrm{kg}$ de poids corporel/jour); $\mathrm{C}=$ concentration de pesticide $(\mu \mathrm{g} / \mathrm{kg}) ; \mathrm{Q}=$ quantité de produits maraîchers consommée (kg/jour); $\mathrm{FC}=$ facteur de correction; $\mathrm{FE}=$ facteur d'exposition ; $\mathrm{P}=$ poids de l'individu.

Dans le cadre de cette étude, le facteur d'exposition (FE) est égal à 1 et représente de ce fait une exposition quotidienne. Aussi, l'estimation a été faite en absence de facteurs de correction (FC) tels que le lavage, l'épluchage, la cuisson, et le broyage qui affectent les niveaux de résidus de pesticide dans les aliments (Claeys et al., 2011). Par ailleurs, sur la base des études réalisées par la direction des statistiques et de la documentation (DSDI) du ministère en charge de l'agriculture de Côte d'ivoire, la proportion maximale de consommation de fruits et légumes en Côte d'Ivoire a été estimée à $5,5 \mathrm{~kg}$ / personne/ an (Fondio et al., 2011). Le poids par défaut pour un adulte était de $60 \mathrm{Kg}$ (Chowdhury et al., 2014). Ainsi, la quantité totale de résidus d'un pesticide donné ingérée par jour et par personne est la somme des teneurs de chaque aliment en résidus de ce pesticide (Son, 2018).

$$
A J E_{\text {total }}=\sum A J E\left(\text { aliment }_{i}\right)
$$

L'évaluation à long terme des risques liés à la consommation de pesticide a été faite à travers l'équation (Lozowicka, 2014) :

$$
\mathrm{HQ}=\frac{A J E}{D J A}
$$

Où $\mathrm{HQ}=$ Quotient de danger $;$ AJE = Apport journalier estimatif de résidus de pesticide; DJA $=$ Dose journalière admissible. 


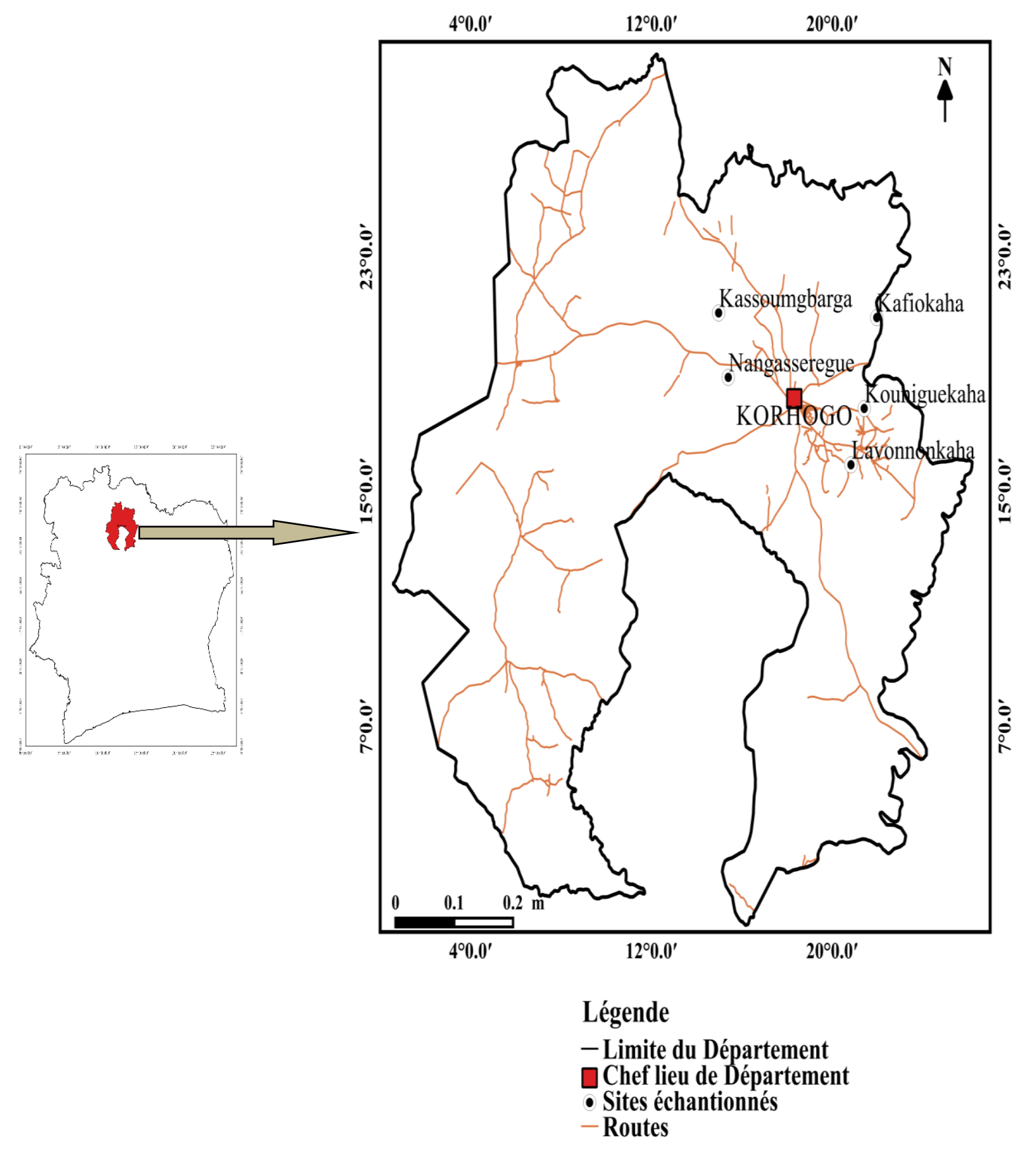

Figure 1: Localisation du département de Korhogo. 


\section{RESULTATS}

\section{Nature et niveau de contamination des produits maraîchers}

L'analyse des produits maraîchers a révélé la présence de résidus de pesticides à savoir le chlorpyriphos éthyle, le cyhalotrine, le cypermethrine, l'ortho-phenylphenol, le parathion, le spirodiclofen, le thiamethoxam et le zoxamid. Ces résidus de pesticides appartiennent aux familles des acides tetroniques, benzamides, neonicotinoïdes, organophosphorés, phénol et pyréthrinoïdes (Tableau 1). Les concentrations maximales en résidu d'ortho-phenylphenol quantifiées au sein des produits maraîchers étaient respectivement de $4,25 \mu \mathrm{g} / \mathrm{kg}$ pour les bulbes d'oignon, de $4,72 \mu \mathrm{g} / \mathrm{kg}$ pour les feuilles d'oignon, de 4,29 $\mu \mathrm{g} / \mathrm{kg}$ pour les laitues, de $4,24 \mu \mathrm{g} / \mathrm{kg}$ pour les piments et de $4,61 \mu \mathrm{g} / \mathrm{kg}$ pour les feuilles de haricot. Le chlorpyriphos éthyle n'a pas été décelé dans les feuilles de haricot et les laitues. En revanche, les plus fortes teneurs en chlorpyriphos éthyle étaient de $1,26 \mu \mathrm{g} / \mathrm{kg}$ pour les bulbes d'oignon, de 0,43 $\mu \mathrm{g} / \mathrm{kg}$ pour les feuilles d'oignon et de 1,34 $\mu \mathrm{g} / \mathrm{kg}$ pour le piment. Concernant le cypermethrine, il n'a été observé que dans les feuilles d'oignon avec une concentration maximale de $0,55 \mu \mathrm{g} / \mathrm{kg}$. Les plus fortes concentrations en résidus de pesticide ont été décelées au sein des feuilles de haricot et ont été estimées à $7,64 \mu \mathrm{g} / \mathrm{kg}$ pour le thiaméthoxame, à $7,33 \mu \mathrm{g} / \mathrm{kg}$ pour le spirodiclofen et à $6,26 \mu \mathrm{g} / \mathrm{kg}$ pour le cyhalotrine.

D'une manière générale, la quantité maximale de chaque résidu de pesticide retrouvée dans les produits maraîchers était en dessous de celle de sa limite maximale préconisée par la commission de régulation européenne. Par ailleurs, l'analyse du Tableau
1 a également montré que tous les produits maraîchers contenaient au moins un (01) résidu de pesticide. En effet, les feuilles d'oignon et les feuilles de haricot contenaient respectivement 7 et 6 matières actives de pesticides contre 3 matières actives pour le piment et 4 matières actives pour les bulbes d'oignon.

\section{Evaluation du risque sanitaire}

Le Tableau 2 présente les apports journaliers en résidus de pesticides fournis par les produits maraîchers pour une personne adulte. L'apport journalier en chlorpyriphos éthyl s'estimait entre $2,68.10^{-5} \mu \mathrm{g} / \mathrm{kg}$ de poids corporel/jour et $31,5.10^{-5} \mu \mathrm{g} / \mathrm{kg}$ de poids corporel/jour. Cet apport en chlorpyriphos éthyle est principalement associé à la consommation de bulbe d'oignon. En ce qui concerne apport journalier en Cyhalotrine, il provenait de la consommation des feuilles de haricot, des feuilles d'oignon et de la laitue et s'estimait entre $14,1 \cdot 10^{-5} \mu \mathrm{g} / \mathrm{kg}$ de poids corporel/jour à $117,3.10^{-5} \mu \mathrm{g} / \mathrm{kg}$ de poids corporel/jour. La consommation journalière de feuille d'oignon de la localité de Kassoumgbarkaha pourrait apporter $3,44.10^{-5}$ $\mu \mathrm{g} / \mathrm{kg}$ de poids corporel/jour de résidus de cypermethrine. Les quantités quotidiennes de résidus d'ortho-phenylphenol ingurgitées lors de la consommation des produits maraichers variaient de $42,9.10^{-5} \mu \mathrm{g} / \mathrm{kg}$ de poids corporel/jour à $106.10^{-5} \mu \mathrm{g} / \mathrm{kg}$ de poids corporel/jour.

Par ailleurs, les quantités de résidus de pesticides apportées suite à la consommation des produits maraîchers ont varié de $3,60.10^{-5}$ $\mu \mathrm{g} / \mathrm{kg}$ de poids corporel/jour à $106.10^{-5} \mu \mathrm{g} / \mathrm{kg}$ de poids corporel/jour pour la localité de Lavonnonkaha, de $3,44.10^{-5} \mu \mathrm{g} / \mathrm{kg}$ de poids corporel/jour à $97,4.10^{-5} \mu \mathrm{g} / \mathrm{kg}$ de poids 
corporel/jour pour la localité de Kassoumgbarkaha, de $6,37.10^{-5} \mu \mathrm{g} / \mathrm{kg}$ de poids corporel/jour à $99,6.10^{-5} \mu \mathrm{g} / \mathrm{kg}$ de poids corporel/jour pour la localité de Kafiokaha, de $8,93.10^{-5} \mu \mathrm{g} / \mathrm{kg}$ de poids corporel/jour à $99,5.10^{-5} \mu \mathrm{g} / \mathrm{kg}$ de poids corporel/jour pour la localité de Kouniguekaha et de $0,74.10^{-5} \mu \mathrm{g} / \mathrm{kg}$ de poids corporel/jour à $117,3.10^{-5} \mu \mathrm{g} / \mathrm{kg}$ de poids corporel/jour pour la localité de Nangasseregue.

De manière générale, en l'absence de facteurs de correction, l'apport journalier estimatif des résidus de pesticides détectés dans les produits maraîchers (bulbe d'oignon, feuilles d'oignon, feuilles de haricot, piment, laitue et tomate) variait entre $0,744.10^{-5} \mu \mathrm{g} / \mathrm{kg}$ de poids corporel/jour et $117,3.10^{-5} \mu \mathrm{g} / \mathrm{kg}$ de poids corporel/jour. La quantité totale de résidus de pesticide qu'un individu peut ingérer quotidiennement est indiquée dans le Tableau 3. Cette quantité équivaut à la somme des teneurs d'un même pesticide contenu dans chaque aliment consommé. L'analyse du Tableau 3 montre que l'apport journalier estimatif (AJE) d'un pesticide pour l'ensemble des aliments consommés varie de 7,4.10-6 $\mu \mathrm{g} / \mathrm{kg}$ de poids corporel/jour à $4350,6.10^{-6}$ $\mu \mathrm{g} / \mathrm{kg}$ de poids corporel/jour.
Une comparaison entre la Dose Jugée Acceptable ou Dose Journalière Admissible (DJA) de résidus de pesticide et l'AJE en résidus de pesticide ingérable pour les aliments (produits maraîchers) a été faite. Cette comparaison avait pour but de déterminer si la consommation de produits alimentaires renfermant des résidus de pesticides conduirait à une absorption de résidus de pesticides supérieurs à la Dose Journalière Admissible (DJA) en vue d'estimer les éventuels effets néfastes sur la santé. Les résultats de cette analyse ont montré que l'apport journalier estimatif en résidus de pesticides est largement inférieur aux différentes Doses Journalières Admissibles (Tableau 3).

Par ailleurs, le ratio de la quantité estimé de résidus ingérable par la dose journalière acceptable était compris entre $0,015 \cdot 10^{-6}$ et $156,6 \cdot 10^{-6}$. Le Zoxamid a enregistré un quotient de danger pratiquement nul $(0,0000)$ pour tous les sites de l'étude. Les quotients de danger les plus élevés ont été observés pour le parathion $\left(156,6 \cdot 10^{-6}\right)$. Dans l'ensemble, le niveau de risque calculé a été jugé acceptable pour l'homme car le quotient de danger ou le ratio de la quantité estimée de résidus de pesticide ingérable par la dose journalière acceptable était très inférieur à 1 . 
Tableau 1 : Variation des teneurs en pesticides des produits maraîchers.

\begin{tabular}{|c|c|c|c|c|c|c|c|c|c|c|c|}
\hline \multirow[t]{2}{*}{ Pesticides } & \multirow[t]{2}{*}{ Famille chimique } & \multicolumn{2}{|c|}{ Bulbe d'oignon } & \multicolumn{2}{|c|}{ Feuille d'oignon } & \multicolumn{2}{|c|}{ Feuille de haricot } & \multicolumn{2}{|l|}{ laitue } & \multicolumn{2}{|l|}{ piment } \\
\hline & & $\begin{array}{l}\text { Teneur } \\
\text { maximale } \\
\text { quantifiée } \\
(\mu \mathrm{g} / \mathrm{kg})\end{array}$ & $\begin{array}{l}\text { LMR } \\
(\mathrm{mg} / \\
\mathrm{kg})\end{array}$ & $\begin{array}{l}\text { Teneur } \\
\text { maximale } \\
\text { quantifiée } \\
(\mu \mathrm{g} / \mathrm{kg})\end{array}$ & $\begin{array}{l}\text { LMR } \\
(\mathrm{mg} / \\
\mathrm{kg})\end{array}$ & $\begin{array}{l}\text { Teneur } \\
\text { maximale } \\
\text { quantifiée } \\
(\mu \mathrm{g} / \mathrm{kg})\end{array}$ & $\begin{array}{l}\text { LMR } \\
(\mathrm{mg} / \\
\mathrm{kg})\end{array}$ & $\begin{array}{l}\text { Teneur } \\
\text { maximale } \\
\text { quantifiée } \\
(\mu \mathrm{g} / \mathrm{kg})\end{array}$ & $\begin{array}{l}\text { LMR } \\
(\mathrm{mg} / \mathrm{kg})\end{array}$ & $\begin{array}{l}\text { Teneur } \\
\text { maximale } \\
\text { quantifiée } \\
(\mu \mathrm{g} / \mathrm{kg})\end{array}$ & $\begin{array}{l}\text { LMR } \\
(\mathrm{mg} / \mathrm{kg})\end{array}$ \\
\hline Chlorpyriphos & Organophosphoré & 1,26 & 0,2 & 0,43 & 0,05 & ND & 0,05 & ND & 0,05 & 1,34 & 0,01 \\
\hline Cyhalotrine & Pyréthrinoïde & ND & 0,2 & 3,09 & 0,5 & 6,26 & 0,5 & 6,11 & 0,5 & ND & 0,1 \\
\hline Cypermethrine & Pyréthrinoïde & ND & & 0,55 & & ND & & ND & & ND & \\
\hline $\begin{array}{l}\text { Ortho- } \\
\text { phenylphenol }\end{array}$ & Phénol & 4,25 & 0,05 & 4,72 & 0,05 & 4,61 & 0,05 & 4,29 & 0,05 & 4,24 & 0,05 \\
\hline Parathion & Organophosphoré & 1,24 & 0,05 & 1,93 & 0,05 & 3,13 & 0,05 & ND & 0,05 & ND & 0,05 \\
\hline Spirodiclofen & Acide tetronique & 1,26 & 0,02 & ND & 0,02 & 7,33 & 0,02 & ND & 0,02 & ND & 0,2 \\
\hline Thiamethoxame & Neonicotinoïde & ND & 0,01 & 3,25 & 0,01 & 7,64 & 0,01 & ND & 5 & ND & 0,7 \\
\hline Zoxamide & Benzamide & ND & 0,02 & 2,02 & 30 & 1,21 & 30 & 0,46 & 30 & 1,07 & 0,02 \\
\hline
\end{tabular}


Tableau 2 : Apports journaliers estimatifs en résidus de pesticides des différents aliments.

\begin{tabular}{|c|c|c|c|c|c|c|c|c|c|}
\hline \multirow[b]{2}{*}{ Localité } & \multirow[b]{2}{*}{ Spéculation } & \multicolumn{8}{|c|}{ Apport Journalier Estimatif en Pesticide ( $\mu \mathrm{g} / \mathrm{kg}$ de poids corporel/jour) } \\
\hline & & Chlorpyrifos & Cyhalotrine & Cypermethrin & OPP & Parathion & Spirodiclofen & Thiamethoxam & zoxamid \\
\hline \multirow{4}{*}{ Lavonnonkaha } & Oignon & $31,5.10^{-5}$ & -2 & - & $106.10^{-5}$ & - & - & - & - \\
\hline & $\begin{array}{l}\text { Feuille (F.) } \\
\text { d'oignon }\end{array}$ & - & - & - & $106.10^{-5}$ & - & - & $27,1.10^{-5}$ & - \\
\hline & F. de haricot & - & $14,1 \cdot 10^{-5}$ & - & $97,2 \cdot 10^{-5}$ & - & - & $44.10^{-5}$ & $3,60.10^{-5}$ \\
\hline & Piment & $11,2.10^{-5}$ & - & - & $82,3 \cdot 10^{-5}$ & - & - & - & - \\
\hline \multirow{5}{*}{ Kassoumgbarkaha } & Oignon & $10,5.10^{-5}$ & - & - & $48,3 \cdot 10^{-5}$ & - & - & - & - \\
\hline & F. d'oignon & - & - & $3,44 \cdot 10^{-5}$ & $97,4 \cdot 10^{-5}$ & - & - & - & $12,6.10^{-5}$ \\
\hline & F. de haricot & - & - & - & $42,9 \cdot 10^{-5}$ & $8,94 \cdot 10^{-5}$ & $53,4 \cdot 10^{-5}$ & - & - \\
\hline & Piment & - & - & - & $81,8 \cdot 10^{-5}$ & - & - & - & - \\
\hline & Laitue & - & - & - & $93,3 \cdot 10^{-5}$ & - & - & - & $5,69.10^{-5}$ \\
\hline \multirow{5}{*}{ Kafiokaha } & Oignon & $16,75 \cdot 10^{-5}$ & - & - & $76.10^{-5}$ & - & - & - & \\
\hline & F. d'oignon & - & - & - & $99,5.10^{-5}$ & - & - & - & $7,31 \cdot 10^{-5}$ \\
\hline & F. de haricot & - & - & - & $89,9.10^{-5}$ & - & - & - & - \\
\hline & Piment & - & - & - & $99,6.10^{-5}$ & - & - & - & - \\
\hline & Tomate & $6,37 \cdot 10^{-5}$ & - & - & $70.10^{-5}$ & - & - & - & - \\
\hline \multirow{4}{*}{ Kouniguekaha } & Oignon & $15.10^{-5}$ & - & - & $75.10^{-5}$ & $14,7.10^{-5}$ & $31,5.10^{-5}$ & - & - \\
\hline & F. d'oignon & - & - & - & $82,8 \cdot 10^{-5}$ & - & - & $17,5 \cdot 10^{-5}$ & - \\
\hline & F. de haricot & - & - & - & $86,2 \cdot 10^{-5}$ & - & - & - & $13,3 \cdot 10^{-5}$ \\
\hline & Piment & - & - & - & $99,5.10^{-5}$ & - & - & - & $8,93 \cdot 10^{-5}$ \\
\hline \multirow{5}{*}{ Nangasseregue } & Oignon & $31,3 \cdot 10^{-5}$ & - & - & $76,3 \cdot 10^{-5}$ & $31.10^{-5}$ & - & - & - \\
\hline & F. d'oignon & $2,68 \cdot 10^{-5}$ & $68,1.10^{-5}$ & - & $79,6.10^{-5}$ & $12,1.10^{-5}$ & - & - & - \\
\hline & F. de haricot & - & $39,1.10^{-5}$ & - & $104.10^{-5}$ & $19,6.10^{-5}$ & - & $47,8 \cdot 10^{-5}$ & $0,74.10^{-5}$ \\
\hline & Laitue & - & $117,3.10^{-5}$ & - & $71,5 \cdot 10^{-5}$ & - & - & - & - \\
\hline & Piment & - & - & - & $99,1.10^{-5}$ & - & - & - & - \\
\hline
\end{tabular}


Tableau 3 : Estimation du risque d'exposition aux résidus de pesticides.

\begin{tabular}{|c|c|c|c|c|c|c|c|c|c|c|c|}
\hline \multirow[t]{2}{*}{ Pesticides } & \multirow{2}{*}{$\begin{array}{l}\text { DJA } \\
\text { ( } \mu \mathrm{g} / \mathrm{kg} / \mathrm{jour})\end{array}$} & \multicolumn{5}{|c|}{ AJE ( $\mu \mathrm{g} / \mathrm{kg}$ de poids corporel/jour) } & \multicolumn{5}{|c|}{ Quotient de danger } \\
\hline & & 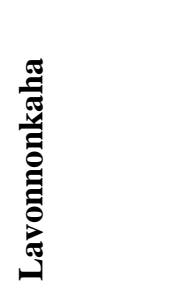 & 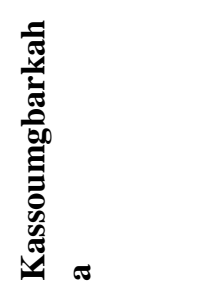 & 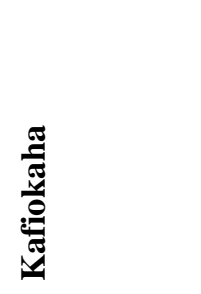 & 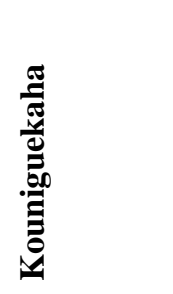 & 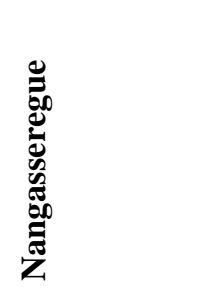 & 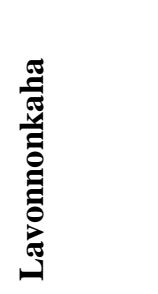 & 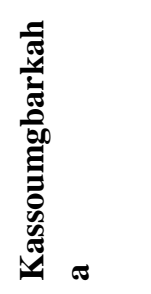 & 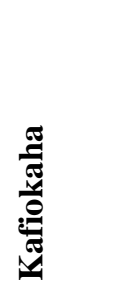 & 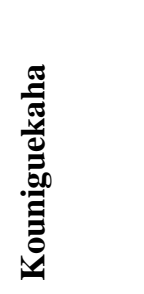 & 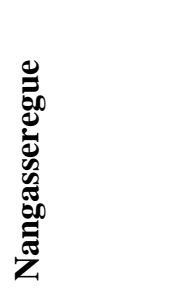 \\
\hline Chlorpyrifos & 10 & $426,8.10^{-6}$ & $105,3.10^{-6}$ & $231,3.10^{-6}$ & $150.10^{-6}$ & $339,3.10^{-6}$ & $42,7.10^{-6}$ & $10,5 \cdot 10^{-6}$ & $\begin{array}{c}23,1.10^{-} \\
6\end{array}$ & $15.10^{-6}$ & $33,9.10^{-6}$ \\
\hline Cyhalotrine & 20 & $141.10^{-6}$ & - & - & - & $2245,2.10^{-6}$ & $7,1.10^{-6}$ & - & - & - & $112,3 \cdot 10^{-6}$ \\
\hline Cypermethrine & $\mathbf{5 0}$ & - & $34,4 \cdot 10^{-6}$ & - & - & - & - & $0,69.10^{-6}$ & - & - & - \\
\hline OPP & 200 & $3921.10^{-6}$ & $3636,9.10^{-6}$ & $4350,6.10^{-6}$ & $3435.10^{-6}$ & $4306,9.10^{-6}$ & $19,6.10^{-6}$ & $18,2.10^{-6}$ & $\begin{array}{c}21,8.10^{-} \\
6\end{array}$ & $17,2.10^{-6}$ & $21,5.10^{-6}$ \\
\hline Parathion & 4 & - & $89,4.10^{-6}$ & - & $147.10^{-6}$ & $626,3.10^{-6}$ & - & $22,4.10^{-6}$ & - & $36,7.10^{-6}$ & $156,6.10^{-6}$ \\
\hline Spirodiclofen & 15 & - & $534,4.10^{-6}$ & - & $315.10^{-6}$ & - & - & $3,6.10^{-6}$ & - & $21.10^{-6}$ & - \\
\hline Thiamethoxam & 26 & $710,8.10^{-6}$ & - & - & $175.10^{-6}$ & $477,5.10^{-6}$ & $27,3 \cdot 10^{-6}$ & - & - & $6,7 \cdot 10^{-6}$ & $18,4.10^{-6}$ \\
\hline Zoxamide & 500 & $36.10^{-6}$ & $183,1.10^{-6}$ & $73,1.10^{-6}$ & $222,3 \cdot 10^{-6}$ & $7,4.10^{-6}$ & $0,07.10^{-6}$ & $0,4 \cdot 10^{-6}$ & $\begin{array}{c}0,15.10^{-} \\
6\end{array}$ & $0,4.10^{-6}$ & $0,015.10^{-6}$ \\
\hline
\end{tabular}




\section{DISCUSSION}

Les produits maraîchers du département de Korhogo sont contaminés par une diversité de pesticides appartenant aux familles chimiques des organophosphorés, des pyréthrinoïdes, des phénols, des acides tetroniques, des neonicotinoïdes et des benzamides. La contamination des produits maraîchers par des pesticides issus de différentes familles chimiques a également été signalée par certains auteurs (Diop, 2013; Pazou-Yehouenou et al., 2013 ; Toure et al., 2015 ; Dakuyo et al., 2020). En effet, la recherche des résidus de pesticides dans les légumes au Bénin par Pazou-Yehouenou et al. (2013) a révélé la présence de 13 pesticides dont le dichlorodiphényltrichloroéthane (DDT), l'endosulfan, l'aldrine, et le lindane. D'autre part, Diop et al. (2013) ont indiqué la présence de 18 pesticides dont les plus prédominants sont le dicofol, le chlorpyrifos, le DDT, le dimethoate et la cyhalothrine dans les échantillons de chou, laitues et tomates de la région des Niayes au Sénégal.

En Côte d'Ivoire, les travaux réalisés par Toure et al. (2015), ont indiqué que la plupart des pesticides retrouvés dans les produits maraîchers de la région du Sud-Est de la Côte d'Ivoire sont la bifenthrine, la deltamethrine et la cyhalotrine tous issus de la famille des pyréthrinoïdes. Kpan et al. (2019) quant à eux ont décelé des résidus de chlorothalonil, de manèbe, de chlorpyrifos éthyle, de carbendazine, de cyperméthrine et de lambdacyhalothrine dans les laitues des sites maraîchers de la commune de Port-Bouët à Abidjan.

Les quantités de pesticides retrouvées dans les produits maraîchers dépendraient non seulement de la composition et de la quantité de produits phytosanitaires pulvérisés, mais également de la teneur en pesticide contenue dans le sol et dans l'eau utilisée pour l'irrigation. En outre, la diversité des pesticides détectée dans les produits maraîchers pourrait s'expliquer probablement par le fait que les cultures maraîchères nécessitent l'utilisation de nombreuses molécules pour lutter contre les insectes, les nématodes et les mauvaises herbes. Les travaux de Coulibaly et al. (2012) corroborent cette hypothèse. En effet, lesdits travaux ont conclu à une utilisation intensive de
13 molécules actives appartenant à 11 familles chimiques dans la région des savanes de la Côte d'Ivoire.

Les analyses ont également indiqué que tous les produits maraîchers contiennent au moins un résidu de pesticide. Ces observations sont en accord avec les conclusions faites par Arienzo et al. (2013). Ils ont montré que 29,6\% des échantillons de légumes étaient contaminés par plus d'un résidu de pesticides. Des différents légumes étudiés, les légumes feuilles présentent le pourcentage de contamination le plus élevé. Cette forte proportion de contamination des légumes feuilles pourrait s'expliquer par la sensibilité de ce type de légume face aux attaques des insectes et pathogènes qui nécessitent l'usage de différentes matières actives pour contrôler ces ravageurs. Bien que contaminé par un éventail de pesticides, avec la présence de 7 matières actives pour les légumes feuilles, la charge maximale de résidus de pesticides observés sur les produits maraîchers n'atteint pas les limites maximales de résidus (LMR) fixés par l'Organisation des Nations unies pour l'alimentation et l'agriculture (FAO) et l'Organisation Mondiale de la Santé (OMS) (EU, 2018). Le niveau de résidu observé en dessous des LMR pourrait indiquer d'une part une éducation des agriculteurs et un usage rationnel des pesticides c'est-à-dire une utilisation en conformité avec les recommandations du fabricant (Bakırcı et al., 2014). D'autre part, cette faible teneur en résidus de pesticides dans les produits maraîchers pourrait être liée à la durée de la période entre l'épandage et l'échantillonnage (Shoiful et al., 2013).

En dépit de leur présence dans les produits maraîchers, les apports journaliers en résidus de pesticides estimés dans le cadre de cette étude étaient 100 fois inférieurs à la dose journalière admissible (DJA). De plus, l'estimation du risque sanitaire a montré que le chlorpyriphos ethyle, le parathion éthyle, le thiaméthoxam, le spirodiclofen, le deltamethrine, le zoxamide, le carbaryl, le cyhalotrine, le cypermethrine et l'orthophenyphenol ne représentaient pas un danger direct pour la santé des consommateurs des produits maraîchers. Des observations similaires ont été faites par Bempah et al. 
(2011) au Ghana, par Claeys et al. (2011) en Belgique et par Osman et al. (2011) en Arabie saoudite. Les travaux réalisés par Bempah et al. (2011) ont indiqué que les pesticides tels que le lindane $\left(\begin{array}{lll}\gamma- & \mathrm{HCH}\end{array}\right)$ et le dichlorodiphényltrichloroéthane (DDT) ne présentaient aucun risque pour la population malgré leurs présences dans les tomates vendues sur les marchés d'accra au Ghana. En effet, l'apport journalier estimatif du $\gamma-\mathrm{HCH}$ et du DDT variait entre $9,14.10^{-6} \mathrm{mg} / \mathrm{kg} /$ jour et $7,68.10^{-4} \mathrm{mg} / \mathrm{kg} / \mathrm{jour}$, et les quotients de danger étaient compris entre $5.10^{-4}$ et 0,26.

En revanche, l'évaluation du risque d'exposition des consommateurs de laitue aux résidus de pesticides dans la commune de PortBouet, Abidjan (Côte d'Ivoire) a relevé que $1,73 \%$ à $97,4 \%$ des consommateurs de laitue sont exposés aux effets chroniques des pesticides (Yao et al., 2016). En outre, l'AJE des pesticides variait entre $0,007 \mathrm{~g} / \mathrm{kg}$ de poids corporel/ jour et $2,254 \mathrm{~g} / \mathrm{kg}$ de poids corporel/ jour. La différence entre les résultats de cette étude et ceux de Yao et al. (2016) pourrait être associée à plusieurs facteurs à savoir : les lieux de production, la qualité des eaux d'irrigation, le non-respect des bonnes pratiques agricoles, les teneurs des résidus de pesticides dans les végétaux et la fréquence de consommation de ces produits (Yao et al., 2016).

Par ailleurs, compte tenu des valeurs de Dose Journalière Admissible (DJA) qui sont généralement plus faibles que celles des doses de référence de l'exposition aigüe (ARfD), l'interprétation des niveaux d'exposition calculés en fonction du pourcentage de ARfD pourrait indiquer l'absence de risque d'exposition aigüe.

\section{Conclusion}

Cette étude a mis en exergue la nature et le niveau de contamination des produits maraîchers par les résidus de pesticides dans le département de Korhogo. En effet, plusieurs résidus de pesticides ont été identifiés dans les produits maraîchers. Ces résidus de pesticides appartiennent aux familles, des organophosphorés, des pyréthrinoïdes, des phénols, des acides tetroniques, des neonicotinoïdes et des benzamides. Les concentrations décelées dans l'oignon variaient de $0,42 \mu \mathrm{g} / \mathrm{kg}$ à $4,25 \mu \mathrm{g} / \mathrm{kg}$. Celles retrouvées dans les piments étaient comprises entre 1,07 $\mu \mathrm{g} / \mathrm{kg}$ et $4,24 \mu \mathrm{g} / \mathrm{kg}$. Pour ce qui est des feuilles de haricot et d'oignon, les concentrations retrouvées ont atteint respectivement 7,64 $\mu \mathrm{g} / \mathrm{kg}$ et $4,72 \mu \mathrm{g} / \mathrm{kg}$. De plus, les concentrations décelées dans les laitues variaient entre $0,46 \mu \mathrm{g} / \mathrm{kg}$ et $6,11 \mu \mathrm{g} / \mathrm{kg}$. Les résidus de pesticides retrouvés sont en dessous des LMR. Les résultats indiquent également qu'en absence de facteurs de correction, l'apport journalier estimatif des résidus de pesticides détectés dans les produits maraîchers a varié de $7,44.10^{-6} \mu \mathrm{g} / \mathrm{kg}$ de poids corporel/jour à $1173.10^{-6} \mu \mathrm{g} / \mathrm{kg}$ de poids corporel/jour. Ainsi, la quantité totale de résidu de pesticide qu'un individu a pu ingérer quotidiennement s'évalue entre $7,4.10^{-6} \mu \mathrm{g} / \mathrm{kg}$ de poids corporel/jour et $4350,6.10^{-6} \mu \mathrm{g} / \mathrm{kg}$ de poids corporel/jour. Le niveau de risque calculé a été jugé acceptable pour l'homme car le quotient de danger est inférieur à 1 . Bien que les quantités de résidus de pesticides ne soient pas jugées nocives pour le consommateur, il n'en demeure pas moins que des efforts restent à fournir pour réduire davantage les concentrations des pesticides, notamment à travers des sensibilisations à l'utilisation des bonnes pratiques phytosanitaires.

\section{CONFLIT D'INTERETS}

Les auteurs déclarent qu'il n'y a aucun conflit d'intérêts.

\section{CONTRIBUTIONS DES AUTEURS}

RIY est l'auteur principal de ce travail et a participé à toutes les étapes de sa réalisation. MJOY, RL, $\mathrm{PK}$ et $\mathrm{VM}$ ont contribué à l'analyse des données et à la rédaction de l'article. $\mathrm{BB}$ a assuré la supervision générale des travaux effectués.

\section{REMERCIEMENTS}

Les auteurs remercient les producteurs maraîchers des sites d'études et les techniciens du laboratoire du Centre Suisse de Recherches Scientifiques pour leur contribution dans la collecte et les analyses des données. Nos remerciements vont aussi à l'endroit des relecteurs anonymes dont leurs critiques et suggestions nous ont permis d'améliorer cet article. 


\section{REFERENCES}

Aloko-N'guessan J, Koffi-Didia MA, Coulibaly HT. 2018. Développement agricole et gouvernance foncière à Tioroniaradougou (Nord de la Côte d'Ivoire). Echo. Géo., 43. DOI: https://doi.org/10.4000/echogeo.15192

Arienzo M, Cataldo D, Ferrara L. 2013. Pesticide residues in fresh-cut vegetables from integrated pest management by ultra performance liquid chromatography coupled to tandem mass spectrometry. Food Control, 31(1): 108-115. DOI: 10.1016/j.foodcont.2012.09.021

Bakırcı GT, Acay DBY, Bakırcı F, Ötles S. 2014. Pesticide residues in fruits and vegetables from the Aegean region, Turkey. Food Chemistry, 160: 379-392. DOI: 10.1016/j.foodchem.2014.02.051

Bempah CK, Donkor A, Yeboah PO, Dubey B, Osei-Fosu P. 2011. A preliminary assessment of consumer's exposure to organochlorine pesticides in fruits and vegetables and the potential health risk in Accra Metropolis, Ghana. Food Chemistry, 128(4): 1058-1065. DOI: 10.1016/j.foodchem.2011.04.013

Chowdhury AZ, Hasan M, Karim N, Fakhruddin ANM, Hossain S, Chowdhury AKM, Akter H, Alam K. 2014. Contamination and Health Risk Assessment of Pesticide Residues in Vegetables from Agricultural Fields of Gazipur District, Bangladesh. AmericanEurasian J. Agric. \& Environ. Sci., 14(5): 421-427. DOI:

10.5829/idosi.aejaes.2014.14.05.12338

Claeys WL, Schmit JF, Bragard C, Guy Maghuin-Rogister G, Pussemier L, Bruno Schiffers B. 2011. Exposure of several Belgian consumer groups to pesticide residues through fresh fruit and vegetable consumption. Food Control, 22: 508-516. DOI: 10.1016/j.foodcont.2010.09.037

Coulibaly L, Coulibaly S, Kamagate B, Sekongo N, Savane I, Gourene G. 2012. Distribution des pesticides d'origine agricole et évaluation de la vulnérabilité des ressources en eaux dans un bassin versant transfrontalier: cas du Comoé, Côte d'Ivoire. European Journal of Scientific Research, 76(4): 601-613.
Dakuyo R, Konate K, Sama H, Sanou A, Kabore K, Diao M, Dibala CI, Dicko MH. 2020. Assessment of onions contamination by pesticides residues and characterization of market gardeners' cultural practices in the region of Boucle du Mouhoun (Burkina Faso). Int. J. Biol. Chem. Sci., 14(9): 3097-3109. DOI: https://dx.doi.org/10.4314/ijbcs.v14i9.10

Diop A. 2013. Diagnostic des pratiques d'utilisation et quantification des pesticides dans la zone des Niayes de Dakar (Sénégal). Thèse de Doctorat, Université du Littoral Côte d'Opale, Dunkerque, $190 \mathrm{p}$.

Dro B, Soro D, Koné MW, Bakayoko A, Kamanzi K. 2013. Evaluation de l'abondance de plantes médicinales utilisées en médecine traditionnelle dans le Nord de la Côte d'Ivoire. Journal of Animal \&Plant Sciences, 17(3): 26312646.

DOI: http://www.m.elewa.org/JAPS/2013/17.3 16

EU (European Union). 2018. Pesticides Database. EU. https://ec.europa.eu/food/plant/pesticides /eu-pesticides-database.

Fondio L, Kouame C, Djidji AH, Traore D. 2011. Caractérisation des systèmes de culture intégrant le gombo dans le maraîchage urbain et périurbain de Bouaké dans le Centre de la Côte d'Ivoire. Int. J. Biol. Chem. Sci., 5(3): 1178-1189.

DOI: 10.4314/ijbcs.v5i3.72251

Kapoor U, Srivastava MK, Srivastava AK, Patel DK, Garg V, Srivastava IP. 2013. Analysis of imidacloprid residues in fruits, vegetables, cereals, fruit juices, and baby foods, and daily intake estimation in and around Lucknow, India. Environmental Toxicology and Chemistry, 32(3): 723-727. DOI: 10.1002/etc. 2104

Kpan kGK, Yao LB, Diemeleou CA, N'guettia RK, Traore SK, Dembele A. 2019. Pratiques phytosanitaires en agriculture périurbaine et contamination des denrées par les pesticides : cas des maraîchers de Port-Bouët (Abidjan). Journal of Animal 
\& Plant Sciences, 41(1): 6847-6863. DOI: 10.35759/JAnmPISci.v41-1.11

Lozowicka B, Kaczynski P, Paritova AE, Kuzembekova GB, Abzhalieva AB, Sarsembayeva NB, Alihan K. 2014. Pesticide residues in grain from Kazakhstan and potential health risks associated with exposure to detected pesticides. Food and Chemical Toxicology, 64: 238-248. DOI: 10.1016/j.fct.2013.11.038

Mambe-Ani P, Ouattara KN, Elleingand FE, Kadjo V. 2019. Assessment of the impact of pesticide use in urban and periurban agriculture in Abidjan, Côte d'Ivoire. Int. J. Biol. Chem. Sci., 13(6): 2824-2837. DOI: $10.4314 /$ ijbcs.v13i6.32

Osman KA, Al-Humaid AI, Al-Rehiayani SM, Al-Redhaiman KN. 2011. Estimated daily intake of pesticide residues exposure by vegetables grown in greenhouses in Al-Qassim region, Saudi Arabia. Food Control, 22: 947-953. DOI: 10.1016/j.foodcont.2010.11.031

Pazou-Yehouenou EA, Soton A, Azocli D, Acakpo H, Lawin H, Fourn L, Fayomi B, Boko M, Houinsa D, Keke JC. 2013. Maraîchage et affections digestives sur le site de Houéyiho en République du Benin. Int. J. Biol. Chem. Sci., 7(5): 19761986. DOI: $10.4314 /$ ijbcs.v7i5.16

RGPH (Recensement général de la population et de l'Habitat), 2014. Principaux résultats préliminaires. RGPH, Côte d'Ivoire, $26 \mathrm{p}$.

Shoiful A, Fujita H, Watanabe I, Honda K. 2013. Concentrations of organochlorine pesticides (OCPs) residues in foodstuffs collected from traditional markets in Indonesia. Chemosphere, 90(5): 17421750.

DOI: 10.1016/j.chemosphere.2012.10.022

SODEXAM. 2014. Données climatiques : zone de Korhogo. Base de données SODEXAM, Côte d'Ivoire, $\mathrm{p} 2$.
Son D. 2018. Analyse des risques liés à l'emploi des pesticides et mesure de la performance de la lutte intégrée en culture de tomate au Burkina Faso. Thèse de Doctorat, Université de liège, Liège, 212 p.

Soro G, Koffi NM, Kone B, Kouakou YE, M'Bra KR, Soro PD, Soro N. 2018. Utilisation de produits phytosanitaires dans le maraîchage autour du barrage d'alimentation en eau potable de la ville de Korhogo (nord de la Côte d'Ivoire) : risques pour la santé publique. Environ. Risque Sante, 17(2): 155-163. DOI: 10.1684/ers.2018.1147

Swarnam TP, Velmurugan A. 2013. Pesticide residues in vegetable samples from the Andaman Islands, India. Environ. Monit. Assess., 185(7): 6119-6127. DOI: 10.1007/s10661-012-3012-3

Toure N, Kouadio KP, Yoboue KE, YaoKouame A. 2015. Évaluation des métaux traces, pesticides et plastifiants dans les produits maraichers (gombo, corète potagère, épinard et aubergine) dans la vallée du Nieki, sud-est de la Côte d'Ivoire. European Scientific Journal, 11(33): 183-196. https://eujournal.org/index.php/esj/article /view/6645

Yao BL, Kpan Kpan GK, Messoum FG, Dembele A, Traore KS. 2016. Évaluation $\mathrm{du}$ risque phytosanitaire lié à la consommation de la laitue (Lactuca sativa) cultivée dans la commune de PortBouët (Abidjan). Rev. Mar. Sci. Agron. Vét., 4(3): 23-30. https://www.agrimaroc.org/index.php/Ac tes_IAVH2/article/view/448

Yapo RI, Mambo V, Alder AC, Ohou-Yao MJ, Ligban R, Dao D, Stamm C, Bonfoh B. 2016. Caractérisation saisonnière des eaux de puits à usage maraîchère et domestique de Korhogo (Côte d'Ivoire). Int. J. Biol. Chem. Sci., 10(3): 1433-1449. DOI: 10.4314/ijbcs.v10i3.41 